\section{IUPAC Organizational Structure Review}

On 11 July 2019, during the IUPAC General Assembly held in Paris, Council approved the motion that a working group be established to undertake a complete review of the organizational structure of IUPAC (C19.3; https://iupac.org/actions-taken-at-iupac-council-andbureau-paris-france-2019/ ). The work has started and the review group will be chaired by Mark Cesa (USA, former IUPAC President and current member of the Finance Committee); additional members of the task group includes Ito Chao (China/Taipei), Michael Droescher (Germany), Lori Ferrins (IYCN) (Australia), and Zhigang Shuai (China/Beijing), and ex officio Javier Garcia-Martinez (Spain, IUPAC Vice President).

The document presented at Council and proposing the review was originally prepared by Richard Hartshorn, IUPAC Secretary General, in March 2019 and is reproduced below.

At the 101st Meeting of the IUPAC Bureau in Bratislava (2018), a break-out session was held in which groups were asked to consider the 2 nd century of IUPAC, and more particularly the questions of what the future might hold and the ways that IUPAC may need to adapt to that future. Groups were asked to consider short, medium, and long term horizons (with subsequent guidance that this might mean 5 years, 15 years, and 50 years into the future). While this session took place right at the end of the Bureau meeting, which meant that there was no time for a full report-back and discussion, one common theme that emerged was the question of whether the IUPAC Divisional structure would be appropriate in the future, particularly given the interdisciplinary approach that is already taken in contemporary research. Indeed, there were questions asked as to whether the structure was particularly appropriate even now. Nearly 20 years ago now, IUPAC radically changed the way that it approached its scientific work, with a shift from work centered on an essentially permanent set of Commissions to work based in a project system, where task groups were established to conduct pieces of work and then dissolved when they were complete. The Divisional structure that had been in place since the early days of IUPAC remained largely unchanged through this change (with the exception of the establishment of the Division of Chemical Nomenclature and Structure Representation, Division
VIII). Instead of running long-term Commissions, Divisions were now tasked with devising and evaluating project proposals, monitoring their progress, and reviewing and approving the scientific output from the projects.

The current structure of IUPAC forms something of a matrix, in which there is a foundation of Divisions, organised on fairly classic sub-discipline lines, with an overlay of Committees, based on interdisciplinary themes that bridge the interests of multiple Divisions. Thus the Committee on Chemistry Education interacts with all of the Divisions in application of the work of education specialists, and similar things can be said of the recently established Interdivisional Committee on Green Chemistry for Sustainable Development. One result of this is that we often have multiple bodies interested in the work of any particular Task Group, which complicates assessment of project proposals, and leads to funding arrangements being more complicated than perhaps they need to be. Once a project is underway, there is sometimes confusion as to which body has oversight of the work, and there is also potential for duplication of effort in maintaining this oversight.

The matrix structure means that IUPAC may have a larger number of sub-groups and committees than it really needs, which then leads to a substantial additional administrative overhead and higher costs for running the organisation. Budgetary complexities also arise as decisions need to be made on how to balance funding for (sub-) disciplines against funding for strategic work in thematic areas.

The Executive Committee has discussed these issues in a recent conference call, during which it also received a report from the Finance Committee on the financial status of the Union. In view of the administrative and financial pressures currently being experienced by IUPAC, the Executive Committee recommends to Bureau and Council that a working party be established to undertake a complete review of the organisational structure of IUPAC. The working party should further be asked to develop proposals for the future organisation of IUPAC, which can be discussed during the 2020-21 biennium, and voted upon at the 2021 Council meeting in Montreal.

For comments and updates, email Mark Cesa <markcesa@comcast.net> https://iupac.org/project/2020-007-1-020 